\title{
Digitalisierung und Nachhaltigkeit
}

\section{Eine Option für das nachhaltige Wirtschaften?}

\begin{abstract}
Um die ökologischen Belastungsgrenzen der Erde nicht zu überschreiten, muss die Wirtschaft im Rahmen der UN-Agenda 2030 und des Pariser Klimaschutzabkommens handeln. Dafür müssen alle Produktions- und Dienstleistungsprozesse neu gedacht und grundlegend umgestaltet werden. Wie kann die Digitalisierung dazu beitragen? Von Sven Klinkow
\end{abstract}

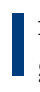
n Deutschland sind diese Ziele in der Nachhaltigkeitsstrategie verankert und im Leitbild der Green Economy formuliert. Die Hightech-Strategie der Bundesregierung nennt nachhaltiges Wirtschaften zudem als prioritäre Zukunftsaufgabe. Wenn Unternehmen vor diesem Hintergrund kohlenstoffarm, energie- und ressourceneffizient sowie umwelt- und sozialverträglich wirtschaften wollen, dann müssen nahezu alle technischen und organisatorischen Produktions- und Dienstleistungsprozesse neu gedacht und grundlegend umgestaltet werden. Dieser Gestaltungsprozess wird aber nur im Zusammenspiel von Wissenschaft, Wirtschaft und Politik gelingen.

Bis Mitte des 18. Jahrhunderts war die begrenzte Verfügbarkeit nutzbarer Energie der limitierende Faktor für Produktion und Weiterverarbeitung von materiellen Gütern, die bis dahin weitestgehend auf menschlicher und tierischer Arbeitskraft sowie auf Wasser- und Windkraft basierten. Erst die Verwendung fossiler Energieträger und die Einführung der Dampfmaschine ermöglichten einen signifikanten technischen Fortschritt und bedeuteten den Beginn der Industrialisierung nach heutigem Verständnis. Fortan revolutionierten Arbeitsmaschinen die Produktions- und Transportmöglichkeiten (früh)industrialisierter Länder, die in Folge mit Elektrifizierung, Computerisierung und Automatisierung weitere industrielle Entwicklungsstufen erreichten. Im 21. Jahrhundert sollen nun digitale Technologien neue Optionen öffnen und zugkräftige Impulse für die Wirtschaft geben. Die Digitalisierung deutet den Beginn einer nächsten industriellen Revolution an (Syska/Lièvre 2016).

Während international die Begriffskombination ,Industrial Internet of Things' (IIoT) etabliert ist, werden in Deutschland zahlreiche Wirtschaftssektoren mit dem Kürzel ,4.0` gekennzeichnet. Prominentes Beispiel ist der Metabegriff ,Industrie 4.0'. Darüber hinaus signalisieren Begriffe wie ,Arbeit', ,Bauen oder , Landwirtschaft ' durch die Erweiterung ,4.0` ihren digitalen Bezug und sollen die Befähigung zur bruchlosen und echtzeit- fähigen Ad-hoc Vernetzung, Kommunikation und Interaktion mittels digitaler Lösungen in den spezifischen Anwendungsfeldern verdeutlichen. Innerhalb dieser meist durch einen hohen Automatisierungs- und Mechanisierungsgrad charakterisierten Branchen wird vorausgesagt, dass es in den nächsten Jahren zu einem umfassenden technologischen Strukturwandel komme, wobei bestehende Produktionsparadigmen infrage gestellt und künftige Produktionsabläufe stark von bestehenden und kommenden digitalen Möglichkeiten determiniert werden. Dabei geht es nicht ausschließlich um Veränderungen in einzelnen Unternehmen, sondern um die grundlegende Neuorganisation industrieller Infrastruktur (Vogel-Heuser 2017).

\section{Digitalisierung und Green Economy}

Entsprechend dem Leitbild der Green Economy könnten digitale Technologien den Gestaltungsprozess zum nachhaltigen Wirtschaften unterstützen und die ökologische Modernisierung der Wirtschaft beschleunigen. Bislang sind die Auswirkungen der Digitalisierung auf Umwelt und Natur weitestgehend noch unbekannt und es wird sehr wahrscheinlich auch zu Nachhaltigkeitskonflikten kommen. So wird allein die $\mathrm{Zu}$ nahme von datenverarbeitenden Geräten den Stromverbrauch deutlich steigern, gleichwohl der Energieverbrauch einzelner Komponenten in den letzten Jahren gesunken ist. Auch wird die Digitalisierung zu erheblichen Veränderungen in der Arbeitswelt führen, mit noch ungeklärten sozialen Auswirkungen. Andererseits öffnen sich durch die Erhebung, Speicherung und Verarbeitung von Daten sowie deren Echtzeitanalyse und -verwertung möglicherweise auch Chancen und neue Optionen für eine nachhaltige Wirtschaftsweise. Dies könnte bestenfalls zur Steigerung der Energie- und Ressourceneffizienz innerhalb produzierender und weiterverarbeitender Branchen führen und ein ,Enabler' für den Klimaschutz sein (Schenk 2014).

Auch wenn noch unklar ist, welche konkreten Chancen sich für Nachhaltigkeit ergeben, besteht ein gewisser Grund zu der Annahme, dass sich mithilfe digitaler Technik die Konkurrenz zwischen ökologischem und ökonomischem Handeln entschärft. So kann die intelligente Vernetzung von Produktionseinheiten einzelne Produktionsprozesse und ganze Wertschöpfungsketten deutlich transparenter machen. Durch die Möglichkeit der kontinuierlichen Erfassung und Auswertung von Zustandsdaten und Produktionsparametern könnten somit frühzeitig Schwachstellen und Risikobereiche erkannt, analysiert und korrigiert werden. Damit wären zumindest Optionen 
für ressourceneffiziente und umweltschonende Produktionsprozesse möglich. Entscheidender Faktor ist hierbei aber ein umweltpolitisch motivierter Gestaltungsprozess, der die Unternehmen zu mehr Mut und Innovationsbereitschaft animiert, um digitale Technik so zu nutzen, dass Klima- und Ressourcenschutz sowie sozial-kulturelle Aspekte in die industrielle Produktion und Fertigung integriert werden.

\section{Herausforderungen in der Umsetzung}

Doch in der Praxis ergeben sich zahlreiche Herausforderungen und Fragen, die bisher noch wenig bis gar nicht untersucht wurden. Aussagen dazu sind oft unspezifisch und beruhen auf wenig konkreten Vermutungen. Grundsätzlich muss daher die Frage gestellt werden, ob sich Ressourcen- und Energieeffizienzpotenziale durch digitale Produktions- und Geschäftsprozesse ergeben und welche ökologischen, ökonomischen und sozial-kulturellen Effekte dabei entstehen. Keinesfalls sind hier ausschließlich positive Auswirkungen zu erwarten, wie die Gefahr von Rebound-Effekten oder die erhöhte Nachfrage nach kritischen Rohstoffen für Hochtechnologieanwendungen zeigen. Eine erfolgreiche Digitale Transformation der Wirtschaft wird sich daran messen lassen müssen, dass nicht nur die technische Umsetzung gelingt, sondern vor allem die einzelnen digital basierten Fertigungs- und Geschäftsprozesse normativen Nachhaltigkeitskriterien entsprechen. Bereits heute lassen sich einige Anwendungsfelder erkennen, in denen digitalisierte Arbeits- und Produktionsprozesse positive Umwelteffekte ermöglichen.

Ein Beispiel hierfür ist die Agrarwirtschaft, die eine global wachsende Bevölkerung mit sich ändernden Ernährungsgewohnheiten auch zukünftig effizient und leistungsfähig versorgen muss. Seit vielen Jahrzehnten ist der Agrarbereich aber auch für erhebliche Umweltprobleme verantwortlich und etwa 14 Prozent der weltweiten THG-Emissionen stammen aus landwirtschaftlichen Produktionsprozessen. Der Einsatz von Düngemitteln sowie die Bodenbearbeitung und Tierhaltung wirken direkt auf Boden, Luft und Wasser. Digitale Technik könnte den bioökonomischen Wertschöpfungsketten der Agrarwirtschaft daher zu einer erheblich besseren Umweltbilanz verhelfen. Beispiele sind automatisierte und teils autonome Landmaschinen, die mittels Stickstoffsensoren über Lichtwellen die Blattfärbung erkennen und direkte Düngeempfehlung geben. In Verbindung mit digitalen Bodenkarten wird die auszubringende Düngermenge der Bodenqualität bedarfsgerecht angepasst und geo-referenziert dokumentiert. Eine boden- und klimaschädliche Überdüngung wird damit vermieden. Die kontinuierliche Erhebung und Analyse von Daten zur Bodenbeschaffenheit in Echtzeit ermöglicht somit die Ad-hocEntscheidungsfindung für den Landwirt, wenn sie nicht gar gänzlich durch die Technik umgesetzt wird. Agrar- bzw. Landwirtschaft 4.0 werden bereits heute in fast jedem fünften Landwirtschaftsbetrieb in Deutschland angewandt, in Betrieben über 100 Hektar sogar in jedem dritten (Bitkom 2016).

\section{Fazit}

Die nächste industrielle Revolution darf nicht nur auf technischer Seite ,revolutionär' sein, sondern muss zugleich eine Transformation zum nachhaltigen Wirtschaften bedeuten. Dabei ist die technische Umsetzung eng mit der Frage nach Innovationskraft und Erfindungsreichtum verknüpft. Bereits jetzt lassen sich zahlreiche Möglichkeiten für nachhaltigkeitseffektive Anwendungslösungen digitaler Techniken in nahezu allen Bereichen der Wirtschaft finden, wie das Beispiel ,Landwirtschaft 4.0` andeutet. Ob diese Lösungen immer den erwarteten und erhofften Erfolg bringen, werden erst intensive Untersuchungen zeigen. Es darf nicht ausgeblendet werden, dass die Digitalisierung auch Negativeffekte hervorbringen kann, wie höhere, und damit effizienzaufzehrende Auslastungsquoten für Maschinen, Fahrzeuge und Infrastruktur, aber auch ethische Fragen bei der Entscheidungsfindung autonom agierender Komponenten. Die Digitalisierung der Wirtschaft sollte deshalb aus der Nachhaltigkeitsperspektive aber nicht bereits grundsätzlich und von vornherein kritisiert werden, sondern mit kreativem Mut nach machbaren ökologischen, ökonomischen und sozial-kulturellen Umsetzungsmöglichkeiten gesucht werden. Eine Chance ist die Digitalisierung daher insbesondere für jene Unternehmen und Wirtschaftszweige, die sich frühzeitig mit den Anforderungen des nachhaltigen Wirtschaftens auseinandersetzen und mit der notwendigen Ernsthaftigkeit mit der Umsetzung beginnen. Dazu gehören weiterhin auch die kritische Auseinandersetzung mit der Digitalisierung im Nachhaltigkeitskontext und der intensive Diskussionsprozess zwischen allen beteiligten Akteuren.

\section{Literatur}

Bitkom (2016): Digitalisierung in der Landwirtschaft. Bitkom, Berlin. Syska, A./Lièvre, P. (2016): Illusion 4.0 - Deutschlands naiver Traum von der smarten Fabrik. Herrieden, CEPTM Publishing.

Vogel-Heuser, B. et al. (2017): Handbuch Industrie 4.0. Bd. 4, 2. Auflage. Berlin, Springer.

Schenk, M. et al. (2014): Fabrikplanung und Fabrikbetrieb - Methoden für die wandlungsfähige, vernetzte und ressourcenintensive Fabrik. Berlin, Springer Vieweg.

\section{AUTOR + KONTAKT}

Sven Klinkow ist wissenschaftlicher Mitarbeiter am Institut für Nachhaltigkeit der HWR Berlin und arbeitet als Wirtschaftsingenieur und Umweltwissenschaftler zu den Themen Digitalisierung, Green Economy und Nachhaltiges Wirtschaften.

Hochschule für Wirtschaft und Recht Berlin, Institut für Nachhaltigkeit, Badensche Straße 50-51, 10825 Berlin

Tel.: +4930308771184, E-Mail: sven.klinkow@hwr-berlin.de

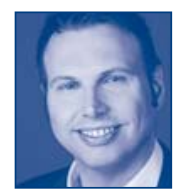

